\title{
Resonant islands without separatrix chaos
}

\author{
G. Corso and F.B. Rizzato \\ Instituto de Física, Universidade Federal do Rio Grande do Sul, Caixa Postal 15051, \\ 91501-970 Porto Alegre Rio Grande Do Sul, Brazil
}

(Received 31 May 1995)

\begin{abstract}
An alternative type of Hamiltonian nonlinear resonant island is analyzed. In the usual case where the resonant island is pendulumlike, chains bifurcated out of the central elliptic point undergo infinite cascades of period-doubling bifurcations as they approach the island boundary. In the present case we find that those chains undergo either period doubling or inverse saddle-node bifurcations, depending on the strength of perturbing terms. In the saddle-node case we show that just after a reconnection process, external chains cross the island boundary to collapse against the bifurcated internal chains.
\end{abstract}

PACS number(s): 05.45.+b

When an integrable Hamiltonian dynamical system described in terms of its action-angle variables, $I$ and $\psi$, is submitted to the action of a nonintegrable perturbation, a vast number of bubbles do appear in the corresponding phase-space. These bubbles, known as the resonant islands of the system, signal positions on the phase-space where the dynamics is particularly sensitive to the perturbation [1].

In the case of one-degree-of-freedom systems, the position of the resonant islands along the action axis can be calculated from the resonance condition

$$
m \omega_{0}\left(I_{m, n}\right)=n \omega,
$$

where $\omega_{0}(I)$ is the frequency of the unperturbed system, $I$ is the corresponding action variable, $\omega$ is the frequency of the perturbing term, and $m, n$ are integers. The frequency $\omega_{0}$, in particular, can be readily obtained as $\omega_{0}=\partial H_{0} / \partial I$ where $H_{0}=H_{0}(I)$ is the integrable part of the complete Hamiltonian $H=H_{0}+H_{1} ; H_{1}$ is assumed to contain the nonintegrable contributions to the dynamics.

In the appropriate set of coordinates, if the amplitudes of the nonintegrable terms are not too large, the phase space in the vicinity of a resonant island can be pictured as follows. One has an elliptic point at the center of the island, a continuum of trapped orbits whose excursions away from the elliptic point are limited by an island boundary and a continuum of untrapped orbits beyond the island boundary with unbounded excursions. It occurs frequently that the boundary contains hyperbolic fixed points. In that case the boundary turns out to be a separatrix with trajectories evolving with zero frequency along it; we shall refer to this type of island as pendulumlike because the corresponding phase-space typically resembles that of a nonlinear pendulum. One can calculate the gyrofrequency $\left(\equiv \omega_{\text {gyro }}\right.$ ) with which the trapped orbits gyrate. What one has to do is to identify the reduced Hamiltonian controlling the dynamics in the vicinity of the island-we shall call it $H_{m, n}$-and to introduce the corresponding appropriate set of action-angle variableswe shall call them $Y$ and $\phi$. Once this is done, the gyrofrequency can be calculated as $\omega_{\text {gyro }}(Y)=\partial H_{m, n} / \partial Y$. The gyrofrequency at the elliptic point, in particular, can be obtained as $\omega_{e} \equiv \omega_{\text {gyro }}(Y \rightarrow 0)$. If the boundary is in fact a separatrix with hyperbolic points along it, one would also have $\omega_{b} \rightarrow 0$, where by $\omega_{b}$ we mean the boundary frequency, $\omega_{b} \equiv \omega_{\text {gyro }}\left(Y \rightarrow Y_{\text {boundary }}\right)$.

Let us now see what happens as the amplitude of the perturbing term start to increase. Whenever

$$
p \omega_{e}=q \omega
$$

with $p, q$ integers, an internal chain of $p$ islands bifurcates out of the elliptic point [2-9]. Then as the perturbation increases in strength, the chain starts to migrate towards the boundary. Considering first the pendulumlike case, at the boundary the density of bifurcated chains is very high. This can be seen if one considers the distance between the actions corresponding to two adjacent $p$ 's, $\left|Y_{p+1}-Y_{p}\right|$, for instance. One has

$$
\left|Y_{p+1}-Y_{p}\right| \sim\left|\frac{\omega_{\text {gyro }}^{2}}{\omega \frac{d \omega_{\text {gyro }}}{d Y}}\right|,
$$

which rapidly goes to zero as $Y \rightarrow Y_{\text {boundary }}$. The proximity of these various chains eventually leads to Chirikov overlap, to infinite cascades of period doubling bifurcation and consequently to chaos [10-13]. Note that according to this picture, chaos first appears in the vicinity of the separatrix; later on it extends toward the innermost regions of the island.

Then, as we turn to the other case, we issue the question motivating the present study. What happens if a particular dynamical system admits a curve separating trapped from untrapped trajectories, which, however, is not a curve along which orbits move with zero frequency? What is going to be the fate of the various bifurcated chains that are born at the elliptic point? Before proceeding to answer the question, we shall see that this situation is of relevance, in particular regarding the physics of particle accelerators; at the same time we shall introduce our model.

Let us assume that a beam of relativistic electrons is submitted to the combined action of a background constant and uniform magnetic field pointing along the $z$ axis of a particular reference frame, and an external electrostatic harmonic wave propagating along the $x$ axis (this model is similar to the one used in Ref. [14]). Then, one 
can write the normalized electronic Hamiltonian, $H$, in the resonant form

$$
H=H_{0}(I)+A_{0} \sum_{l=-\infty}^{+\infty} J_{l}(k \sqrt{2 I}) \cos (l \psi-\omega t)
$$

where the wave amplitude $A_{0}$ measures the intensity of the nonintegrable term, $H_{0}=\left[1+2 I+P_{z}^{2}\right]^{1 / 2}, P_{z}$ is the electron momentum along the background field, $J_{l}$ 's are Bessel functions, and $k$ is the wave vector. The Hamiltonian (3) generates a set of resonant actions that can be located according to relation (1). Not all the resonances generated by relation (1) are directly seen from the Hamiltonian, though; those with $n \neq \pm 1$ can be obtained only in higher perturbative orders with the use of Lie perturbative theories [5]. We shall however consider all the resonances obtained from relation (1) on the same footing to distinguish them from the internal resonances obtained from relation (2). Resonances obtained from Eq. (1) shall be therefore called external and shall be denoted with square brackets as $[m, n]$; internal resonances shall be denoted by the symbol $(p, q)$.

If a positive root $I_{m, n}$ is far above $I=0$ and $A_{0}$ is small enough, one can take $I \sim I_{m, n}=$ const in the wave term and the resonant orbits can be approximately described in terms of a nonlinear pendulum $[1,3]$. On the other hand, if $I_{m, n}$ approaches zero, the dependence of the wave term on the action cannot be approximated as above and the orbit ceases to be pendulumlike. Can one easily encounter situations where $I_{m, n}$ does indeed vanish? The answer is positive. What is usually done in an accelerating system, for instance, is precisely to adjust the wave frequency in such a way as to match the frequency $\omega_{0}(I \rightarrow 0)$ associated with the low-energy particles to be accelerated [14] [from Eq. (3) it is seen that the lower the energy, the smaller $I]$. Keeping these observations in perspective, from now on we set a lowenergy cyclotron resonance condition with $\omega=1$ and $P_{z}=0$, which effectively defines our lowest-energy resonance as $I_{1,1}=0$. We will see that bifurcations in this case, $P_{z}=0$, seem to present a larger variety of behaviors than in cases with $P_{z}>0$ studied previously [9].

Let us then analyze the lowest-energy island engendered by the $[1,1]$ resonance; we will find it to be very unpendulumlike. To carry out the analysis we discard all other resonances from the Hamiltonian (3), perform a time removal canonical transformation

$$
\begin{gathered}
\psi^{\prime}=\psi-t, \\
I^{\prime}=I,
\end{gathered}
$$

and

$$
H^{\prime}=H-t,
$$

recall that $I \ll 1$, and write the resulting resonant Hamiltonian (primes dropped) in the form

$$
H_{1,1}=-\frac{1}{2} I^{2}+A_{0} \frac{\sqrt{2 I}}{2} \cos \psi
$$

where, besides $\omega=1$, we have also set $k=1$. The maximum amplitude of the island is obtained by setting $\psi=H_{1,1}=0$ in Eq. (5); condition $H_{1,1}=0$, in particular, defines the boundary trajectory; i.e., the trajectory dividing trapped from untrapped orbits in the $(I, \psi)$ phase-space. The maximum amplitude can thus be evaluated as $I_{\max }=2^{1 / 3} A_{0}^{2 / 3}$.

Now we come to our point. The Hamiltonian (5) also yields the following remarkable results: the $[1,1]$ island contains only one elliptic fixed point located within it at coordinates $\psi_{e}=0$ and $I_{e}=(1 / 2) A_{0}^{2 / 3}$. In contrast to the pendulumlike case, however, there are no hyperbolic points along the island boundary. This means that, although the boundary separates distinct regions of the phase-space, it is not a zero-frequency trajectory (contour levels of $H_{1,1}$ are displayed in Fig. 1). The model is thus seen to provide a simple and relevant example of the kind of situation we wish to study.

As commented upon earlier, there are two natural frequencies connected with a resonant island, $\omega_{e}$ and $\omega_{b}$. We can obtain expressions for these two frequencies. Frequency $\omega_{e}$ can be readily obtained if one quadratically expands the Hamiltonian near the elliptic point; it reads $\omega_{e}=0.866 A_{0}^{2 / 3}$. Frequency $\omega_{b}$ can be cast in the following form if one takes into account the canonical equations produced by Eq. (5), the fact that $H_{1,1}=0.0$ on the boundary, and the fact that the boundary orbit spends a vanishingly small time flying from points $A$ to $B$ of Fig. 1 (this last feature follows from the fact that $d \psi / d t \sim 1 / \sqrt{I}$, as $I \rightarrow 0)$ :

$$
\omega_{b}=\frac{\pi A_{0}^{2 / 3}}{4\left(\int_{0}^{1} d x \frac{1}{\sqrt{1-x^{6}}}\right)}=0.763 A_{0}^{2 / 3},
$$

where $x^{3}=I^{3} /\left(2 A_{0}^{2}\right)$. Note that points $A$ and $B$ of Fig. 1 are fixed. Apart from that, they seem to play no crucial role in the boundary dynamics, because trajectories reaching or exiting them, do that in a finite amount of time; these points are not hyperbolic. When we speak, as above, of the fly time from $A$ to $B$, we really mean the fly time from the neighborhood of $A$ to the neighborhood

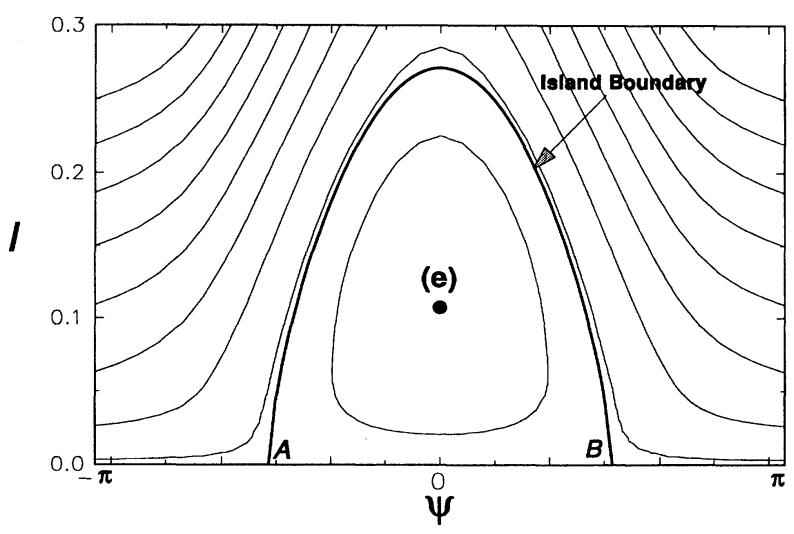

FIG. 1. Contour levels of $H_{1,1}$. We take $A_{0}=0.1$. The island boundary is highlighted. The central elliptic point is denoted as (e). 
of $B$.

Both quantities, $\omega_{e}$ and $\omega_{b}$, are plotted in Fig. 2 as a function of $A_{0}$. It is to be remarked that the elliptic fixed point frequency is always larger than the boundary frequency.

We are now in a position to conjecture on what happens with chains bifurcating out of the elliptic central fixed point. As one increases the wave amplitude from small values, a condition like Eq. (2) is eventually satisfied with $p, q$ integers. As said, whenever this happens, a chain of $p$ islands [with winding $(q / p)$ ] bifurcates out of the elliptic point. Further increase on $A_{0}$ causes the chain to drift towards the island boundary where the frequencies are smaller than in the central region and where, therefore, the winding number can be maintained. This is what is being represented by the horizontal lines of Fig. 2. The horizontal line on the left represents an exemplifying $(7,1)$ chain and the one on the right represents a $(7,3)$ chain. At the left end of each line, the chain is created and at the right end it tentatively arrives at the boundary. In order to see what may happen with the chain during its travel towards the boundary, let us examine the dynamics according to the strength of the wave amplitude.

If during the tentative lifetime of a $(p, q)$ chain between the end points of its horizontal line the amplitude of the perturbing term attains too large a value, the width of the island chains will be also relatively large, overlap with neighboring chains will be a possibility, and the island will likely undergo period doubling bifurcations before it reaches the boundary. This case will be thus very similar to the one occurring in pendulumlike islands where internal chains always overlap (and undergo period doubling) as they near the separatrix. But on the other hand, if during the lifetime of a $(p, q)$ chain the amplitude of the perturbing term remains very low, the overlap possibility will be reduced and the chain may reach the boundary untouched.

Without entering into the detailed calculations, it turns out that a critical perturbing amplitude exists such that one can really speak in terms of relatively "large" and "small" perturbing strengths; it has been calculated in Ref. [7]. The calculation has shown that resonance

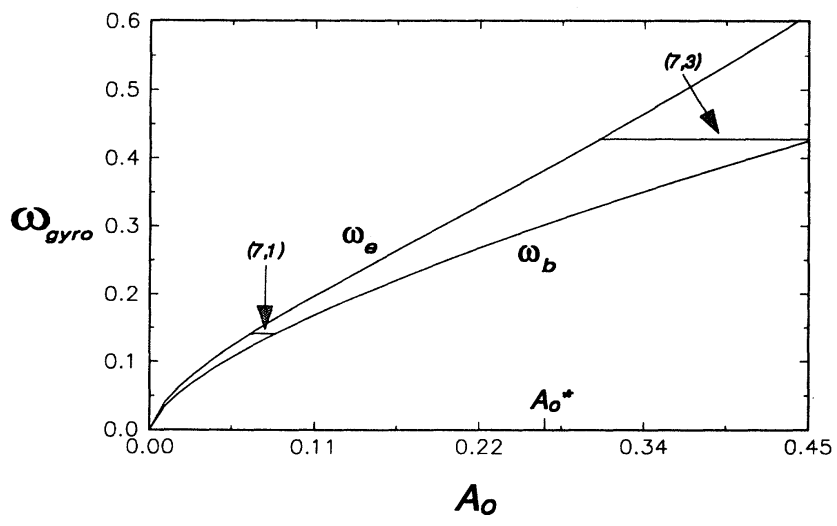

FIG. 2. Functions $\omega_{e}$ and $\omega_{b}$, and chains $(7,1)$ and $(7,3)$. $A_{0}^{*}$ is the estimated period doubling threshold. overlaps within a $[1,1]$ island like the one we are presently studying start to happen when chains with $p_{\sim}^{<3}$ and $q=1$ are created close to the elliptic fixed point. This corresponds to fields of intensity $A_{0} \sim 0.27$. For larger values of $p$, which correspond to smaller values of $A_{0}$, resonance overlaps are absent. In Fig. 2, this particular value of $A_{0}$, which we call $A_{0}^{*}$, falls more or less on the middle of the horizontal axis. This means that the leftmost chain shown in the figure should be expected to arrive at the boundary without undergoing period doubling; the other chain, on the other hand, should be expected to undergo period doubling somewhere before arriving there. The quantity $A_{0}^{*}$ can be thus seen as being a threshold value for period doubling bifurcations.

Then we come back to the final question not yet answered: What happens with a chain that arrives at the separatrix without undergoing period doubling; how can it then vanish? The answer, to be checked against the simulations coming shortly, is that in this case the island collapses against an external chain that reaches the boundary simultaneously. This is not difficult to accept as reasonable. In fact, as the internal chain with winding $q / p$ reaches the boundary, an external chain with winding $n / m=(p-q) / p$ also arrives there (the reason for the $p-q$ factor is that when one performs the time re-
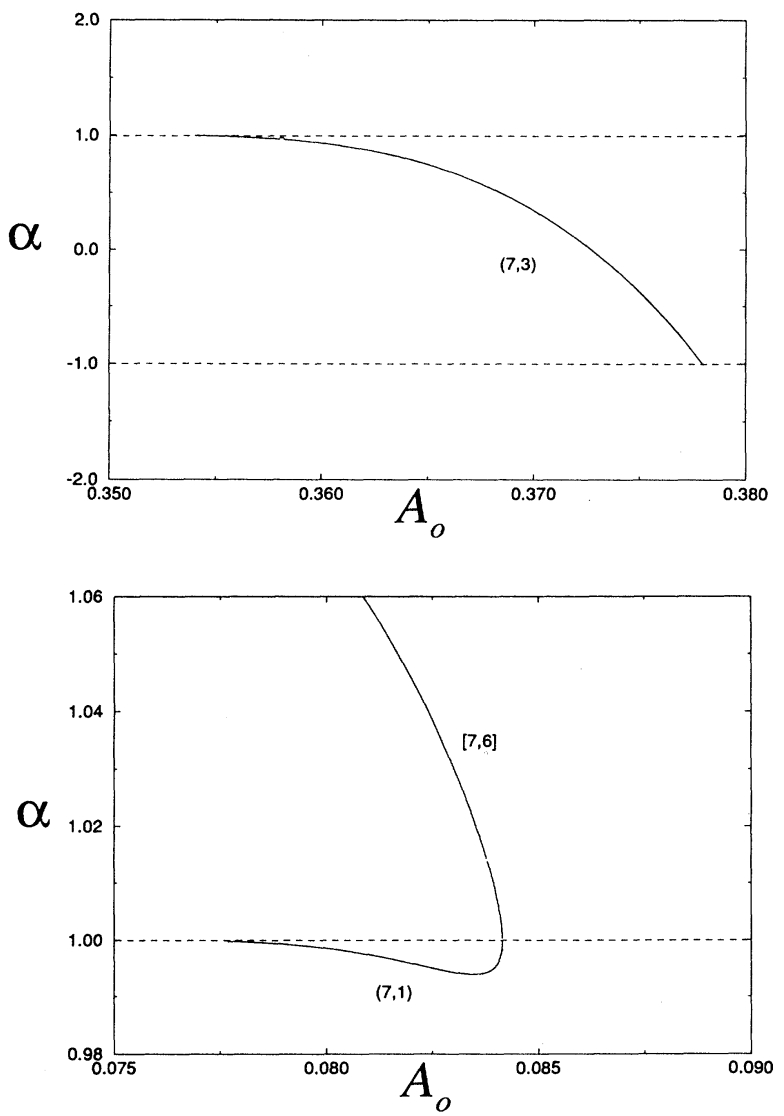

FIG. 3. Stability index $(\alpha)$ for internal chains $(7,3)$ in (a) and $(7,1)$ in $(b)$. The internal $(7,1)$ chain is shown to collapse against an external $[7,6]$ chain via an inverse saddle-node bifurcation. 
moval canonical transformation, all the windings have a $p / p$ factor subtracted; the remaining negative signal is explained because it can be shown that internal orbits gyrate counterclockwise, while $d \psi / d t>0$ ). Let us then turn to numerics in order to check and improve the picture we have so far.

The first case to be analyzed is the $(7,3)$ chain, the one on the right-hand side of Fig. 2. Let us accompany the stability index $\alpha$ of its elliptic fixed points. We recall that if $\alpha$ crosses the line $\alpha=+1$ the island undergoes inverse saddle-node bifurcation, and if $\alpha$ crosses the line $\alpha=-1$ the chain undergoes period doubling bifurcation [8]. As seen in Fig. 3(a) the chain undergoes the usual period doubling bifurcation. This is in agreement with our estimates; the amplitude $A_{0}$ in this case is so large that it is well above the estimated period doubling threshold $A_{0}^{*}$. Let us examine the other case. In this situation $A_{0}$ is well below $A_{0}^{*}$ during the whole existence of the chain. Accordingly, Fig. 3(b) shows that the island un-
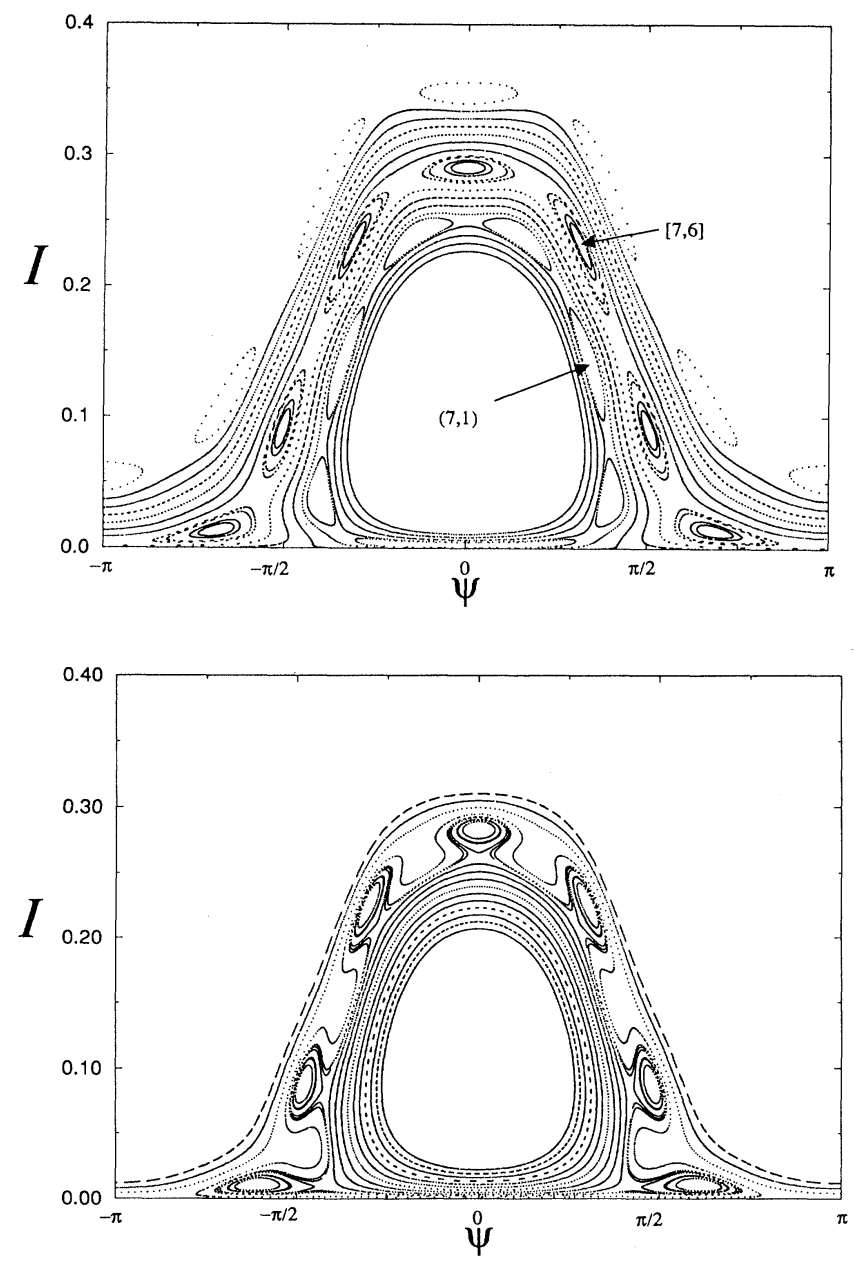

FIG. 4. Details of the inverse saddle bifurcation of the $(7,1)$ chain. Note the presence of the external $[7,6]$ chain just beyond the boundary in (a) $\left(A_{0}=0.0832\right)$ and how it is partially absorbed by the resonant island in (b) $\left(A_{0}=0.0841\right)$. For slightly larger values of $A_{0}$, the chains undergo the inverse saddle-node bifurcation. dergoes inverse saddle-node bifurcation. Moreover, the bifurcation occurs as the internal chain collapses against an external $[7,6]$ chain.

The reader is now referred to Fig. 4 for more details about this latter case. In Fig. 4(a) are shown the $(7,1)$ and $[7,6]$ chains just after the bifurcation, and from Fig. 4(b) we can see how the bifurcation is processed. First, there occurs a reconnection process where the elliptic fixed points of the internal and external chains interchange places. The internal elliptic points are incorporated into the external chain and the external elliptic points into the internal chain. The impression one has is that the external chain crosses the boundary; in a sense, only half of the external island does that. Later on, as the amplitude $A_{0}$ is increased, the elliptic and hyperbolic fixed points of both pairs of "bound-states" thus formed collapse against each other via the inverse saddle-node bifurcation. We have pursued some other chains observing that the process is not merely accidental; $(p, q)$ chains with relatively large (small) $q$ 's tend to undergo period doubling (inverse saddle node). An index known as the Poincaré index [4] is conserved during the process. The conservation of this index must occur for this kind of system and it means that stable and unstable points must be created or destroyed in pairs.

The series of reconnections and saddle-node bifurcations observed in the present work can be related to results obtained in previous papers $[9,15-18]$. To see this, we first point out that the gyrofrequency of our system can be regarded as displaying a nonmonotonic character. In fact, as one moves away from the elliptic fixed point towards the $[1,1]$ island boundary, the frequency of trapped orbits decreases, as we have already seen; then, as one crosses the boundary and starts to move up the phase-space, the frequency starts to increase again. This frequency growth outside the island can be understood in view of the fact that when we speak of frequencies, we really mean frequencies measured relatively to the wave frequency (= 1 in our case); we had introduced this "frame" transformation [Eq. (4)] to define the whole dynamics within the $[1,1]$ island, in particular to define $\omega_{c}$ and $\omega_{b}$, and we have to be consistent in all the following calculations. This amounts to saying that the effective frequency without the central island can be appoximated by $1 / \sqrt{1+2 I}-1$ if one is far above that island, a result indeed indicating frequency growth in absolute values as $I \rightarrow \infty$. Now, as pointed out earlier in the text, the boundary arises as a common curve along which the decreasing internal frequency and the increasing external frequency assume equal values, and as analyzed in the above mentioned papers $[9,15-18]$, the associated nonmonotonic character of the frequency ultimately leads to reconnections and saddle nodes at this location. In contrast to the work of Van der Weele et al. [16], however, the reconnections arising in the Hamiltonian model we used occur not only for one-third resonances.

To summarize, we have analyzed a different type of resonant island where the boundary is free from separatrix chaos. The absence occurs by virtue of the fact that, unlike the pendulumlike case, the boundary of this type of island is in fact free from the presence of hyperbolic 
unstable points. Chains that bifurcate out of the central elliptic point of the resultant kind of island have some unusual behavior. We have shown that a critical perturbing strength exists $\left(A_{0}^{*}\right)$ above which the chains undergo infinite cascades of period doubling bifurcation (in this case the behavior is similar to the one seen in pendulumlike resonances) and below which the chains undergo inverse saddle-node bifurcations as they collapse against external chains at the island boundary - for other values of $P_{z}$, like $P_{z}=1$ [9], the bifurcational behavior seems to be dominated by the saddle-node bifurcations only. The external chains were shown to be partially absorbed by the resonant island before collapse. We feel that this kind of island is of relevance in problems involving nonlinear dynamics. In particular, an accelerating system frequently deals with particles whose low initial energy can easily set them within the domain of the islands.

We are indebted to Iberê Caldas, Arthur Lopes, and Darcy Dillenburg for the useful discussions we had on the subject of Hamiltonian bifurcations. We acknowledge partial support by CNPq and FINEP (Brazil). Part of the numerical work was performed on the Cray YMP. $2 \mathrm{E}$ at the Supercomputing Center of the Universidade Federal do Rio Grande do Sul.
[1] A. J. Lichtenberg and M. A. Lieberman, Regular and Stochastic Motion (Springer, Berlin, 1983).

[2] A. Fukuyama, H. Momota, R. Itatani, and T. Takizuka, Phys. Rev. Lett. 28, 701 (1977).

[3] B. Chirikov, Phys. Rep. 52, 263 (1979).

[4] D.K. Arrowsmith and C.M. Place, An Introduction to Dynamical Systems (Cambridge University Press, Cambridge, England, 1990).

[5] J. Candy and W. Rozmus, Physica D 52, 267 (1991).

[6] Y. Nomura, Y.H. Ichikawa, and W. Horton, Phys. Rev. A 45, 1103 (1992).

[7] G. Corso and F. B. Rizzato, J. Plasma Phys. 49, 425 (1993).

[8] G. Polymilis and K. Hyzanidis, Phys. Rev. E 47, 4381 (1993).

[9] G. Corso and F. B. Rizzato, Physica D 80, 296 (1995).
[10] T. C. Bountis, Physica D 3, 577 (1981).

[11] G. Constopoulos, Physica D 7, 142 (1983).

[12] J. M. Greene, R. S. MacKay, F. Vivaldi, and M. J. Feigenbaum, Physica D 3, 468 (1989).

[13] E. Ott, Chaos in Dynamical Systems (Cambridge University Press, Cambridge, England, 1993).

[14] R. C. Davidson, Y.-T. Yang, and R. E. Aamodt, J. Plasma Phys. 41, 405 (1989).

[15] J. E. Howard and S. M. Hohs, Phys. Rev. A 29, 418 (1984).

[16] J. P. Van der Weele, T. P. Valkering, H. W. Chapel, and T. Post, Physica A 153, 283 (1988).

[17] J. E. Howard and J. Humpherys, Physica D 80, 256 (1995).

[18] G.A. Oda and I. Caldas, Chaos, Solitons, Fractals 5, 15 (1995). 